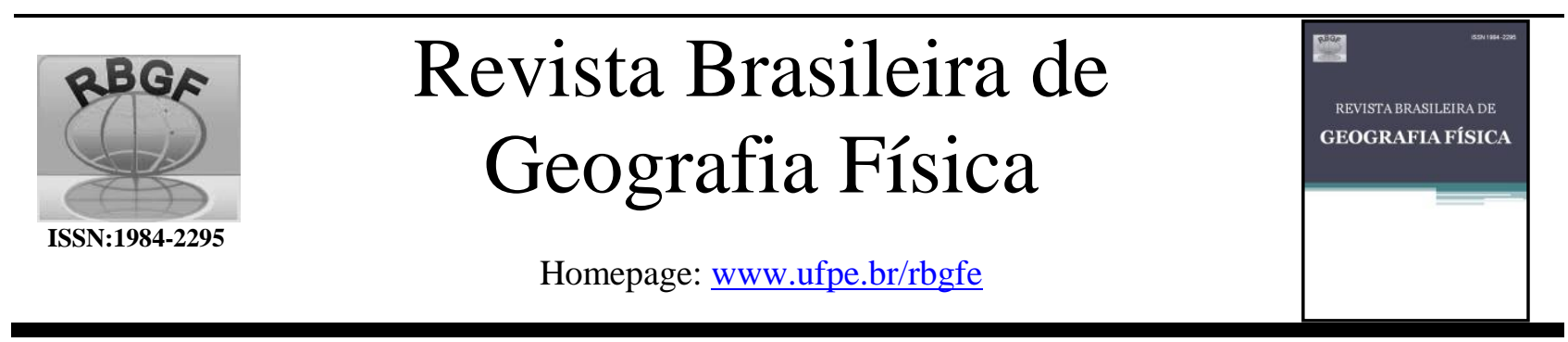

\title{
Análise de Modelos Digitais de Elevação e geração de atributos topográficos para a região da Serra do Mar do estado de Santa Catarina
}

\author{
Yara Rúbia de Melloํㅜ Claudinei Taborda da Silveira $^{2}$
}

\begin{abstract}
${ }^{1}$ Doutoranda em Geografia, Programa de Pós-Graduação em Geografia, Universidade Federal do Paraná, Av. Cel. Francisco H. dos Santos, 100, Ed. João José Bigarella, $1^{\circ}$ andar, sala 108, Jardim das Américas, CEP 81531-980, Curitiba, Paraná. (41) 3361-3450. Autor corresponde: yarademello@gmail.com (autor correspondente). ${ }^{2}$ Dr. Professor, Programa de Pós-Graduação em Geografia, Universidade Federal do Paraná, Av. Cel. Francisco H. dos Santos, 100, Ed. João José Bigarella, $1^{\circ}$ andar, sala 108, Jardim das Américas, CEP 81531-980, Curitiba, Paraná. (41) 3361-3450. claudinei.ufpr@yahoo.com.br.
\end{abstract}

Artigo recebido em 09/11/2014 e aceito em 24/02/2015

\section{R E S U M O}

A análise geomorfométrica consiste na quantificação morfológica da superfície terrestre com enfoque na extração de parâmetros calculados de Modelos Digitais de Elevação (MDEs), também compreendida como análise digital do relevo. A região da Serra do Mar de Santa Catarina apresenta uma diversidade de formas de relevo e existem distintos MDEs disponíveis para a região, desde modelos globais até base de dados no âmbito estadual. Neste contexto, o objetivo do trabalho foi comparar diferentes MDEs e com o modelo mais adequado realizar uma caracterização geomorfométrica da região da Serra do Mar de Santa Catarina. Para tanto, foi delimitado um polígono englobando a Serra do Mar do Estado e áreas adjacentes, dentro do qual foi selecionado um polígono menor como área teste. Foram selecionados os modelos globais SRTM90, SRTM30, Aster30, Topodata e o MDE do Levantamento Aerofotogramétrico de Santa Catarina (resolução $=1 \mathrm{~m}$ ) - que foi reamostrado para as resoluções de 5 e 10 metros; a avaliação dos modelos se deu por meio da geração de atributos topográficos na área teste, conferências in loco e utilização de pontos de controle. Posteriormente, foram gerados os atributos topográficos: hipsometria, declividade, aspecto, plano de curvatura, perfil de curvatura e relevo sombreado; e do Índice de Posição Topográfica, para todo o polígono de área de estudo com o MDE que apresentou o melhor desempenho. O MDE de Santa Catarina foi o modelo que se mostrou mais adequado com base nas análises. Os MDEs globais apresentaram resultados insatisfatórios em relação a atributos topográficos como o aspecto e a declividade. Palavras-chave: Relevo, Aspecto, Índice de Posição Topográfica.

\section{Analysis of Digital Elevation Models and generation of topographic attributes for the Serra do Mar region of the state of Santa Catarina}

\begin{abstract}
A B S T R A C T
The geomorphometric analysis consists of the quantification of the terrestrial surface with focus on the extraction of calculated parameters of Digital Elevation Models (DEMs), also understood as digital analysis of the relief. The Serra do Mar region of Santa Catarina presents a diversity of relief forms and there are distinct DEMs available to the region, from global models to a State model. In this context, the goal of this study was to compare different DEMs and with the most adequate model perform a geomorphometric characterization of the Serra do Mar region of Santa Catarina. For this purpose, a polygon was delineated, encompassing the Serra do Mar of Santa Catarina and adjacent areas, within which a smaller polygon was selected as the test area. The global models SRTM90, SRTM30, Aster30, Topodata and the DEM of the Aerophotogrammetric Survey of Santa Catarina (resolution $=1 \mathrm{~m}$ ) - that were exported to resolutions of 5 and 10 meters, were selected; the evaluation of the models occurred through the generation of topographic attributes in the test area, on-site conferences and use of control points. Afterwards, maps of the topographic attributes were elaborated: hypsometry, slope, aspect, plane of curvature, profile of curvature and hillshade; and the Topographic Position Index, for the entire polygon of study area with the DEM that presented the best performance. The DEM of Santa Catarina was the most appropriate model based on the analyzes. The global DEMs presented unsatisfactory results in relation to topographic attributes such as aspect and slope.
\end{abstract}

Keywords: Relief, Aspect, Topographic Position Index. 


\section{Introdução}

A Geomorfometria é atualmente definida como a ciência das análises quantitativas da superfície terrestre (Pike, 2000) e têm como essência a automação da extração de informações a partir de Modelos Digitais de Elevação - MDE. É uma ciência interdisciplinar, pois envolve diferentes campos do conhecimento, como as Ciências da Terra, a Matemática e mais recentemente a Ciência da Computação (Hengl et al., 2009).

O foco operacional da Geomorfometria é a extração de parâmetros e objetos da superfície terrestre. Os parâmetros são referenciados na literatura de diferentes formas, como atributos ou propriedades topográficas, parâmetros de formas do relevo, variáveis morfométricas, informações do terreno, atributos do terreno e atributos geomorfométricos (Hengl et al., 2009). São exemplos de atributos topográficos (designação adotada neste estudo) a declividade, a orientação da vertente e o relevo sombreado; e de objetos, bacias hidrográficas, dunas de areia e montanhas.

Na segunda metade do século XX, com os avanços tecnológicos impulsionados pelas grandes guerras mundiais, a Geomorfometria passou a estar intimamente relacionada à computação e ao sensoriamento remoto, resultando em uma moderna abordagem analítica cartográfica.

Em acordo com Silva et al. (2017a) a geomorfometria têm se constituído com um arcabouço metodológico imprescindível, em destaque no campo da Geografia Física, sendo que de maneira evolutiva, vem contribuído em muitas pesquisas que utilizam a informação topográfica como premissa.

Os MDEs são muito utilizados nas análises geomorfométricas, pois possuem coberturas contínuas de áreas extensas e podem ser trabalhados de modo automatizado em softwares específicos. Essas ferramentas vêm se tornando cada vez mais acessíveis, precisas e robustas (Hengl et al., 2009; Silveira \& Silveira, 2015).

MDEs vêm sendo utilizados em distintos trabalhos relacionados a análises ambientais. Tais como, estudos relacionados ao mapeamento digital de solos (Silveira et al., 2013; Nowatzki et al., 2017); análises de bacias hidrográficas (Fan et al., 2013; Trentin et al, 2015); identificação de suscetibilidade a processos movimentos gravitacionais em encostas (Silveira et al, 2013 e 2014; Pozzobon et al., 2015); e também na classificação e compartimentação de formas de relevo (De Reu et al., 2013; Guadagnin \& Trentin,
2014; Tinós et al., 2014; Silveira et al., 2014, 2016 e 2017; Silva et al., 2017b; Gomes et al., 2018).

A Serra do Mar é uma formação montanhosa que se estende por aproximadamente $1.000 \mathrm{~km}$ pela costa brasileira, desde o estado do Rio de Janeiro até o nordeste do estado de Santa Catarina. No nordeste de Santa Catarina, as formas do relevo podem ser caracterizadas em três grandes compartimentos: planalto a oeste, Serra do Mar na porção central, e planície costeira a leste. O planalto compreende extensa área, onde predominam pequenas cristas, morros de topos convexos e vales em "V" e simétricos; a escarpa da Serra do Mar é formada por corpos graníticos intrusivos e vales simétricos em forma de "V", caracterizada ainda por altas declividades e atinge altitudes superiores a 1.500 metros em algumas áreas; por fim, a planície costeira abrange áreas de baixas altitudes - inferiores a 100 metros nas planícies aluviais até o entorno da Baía da Babitonga e contém ainda morros e morretes de topos convexos (Oliveira, 2006).

Tendo em vista a dinâmica do relevo regional e a disponibilidade de MDEs para caracterizar a região, buscou-se com este estudo analisar distintos MDEs, com diferentes características de aquisição e processamento desde modelos globais até um modelo estadual, e com o modelo mais adequado realizar uma caracterização geomorfométrica da região da Serra do Mar de Santa Catarina.

\section{Material e métodos}

Área de Estudo

A área de estudo compreende parte da região nordeste do estado de Santa Catarina, conforme polígono destacado na Figura 1, o qual engloba a Serra do Mar e áreas de planície e planalto adjacentes. Por ter sido utilizado um polígono, foram consideradas áreas do território paranaense, porém o foco da análise se dá em Santa Catarina. O polígono encontra-se entre as latitudes de $25^{\circ} 57^{\prime} 09^{\prime \prime S}$ e $26^{\circ} 31^{\prime} 09^{\prime \prime} \mathrm{S}$, e longitudes de $49^{\circ} 37^{\prime} 56^{\prime \prime S}$ e $48^{\circ} 29^{\prime} 00^{\prime \prime S}$. Com área total de $7.195,2 \mathrm{~km}^{2}$, perímetro de $354,7 \mathrm{~km}$, extensão latitudinal de $63 \mathrm{~km}$ e longitudinal de $115 \mathrm{~km}$. 


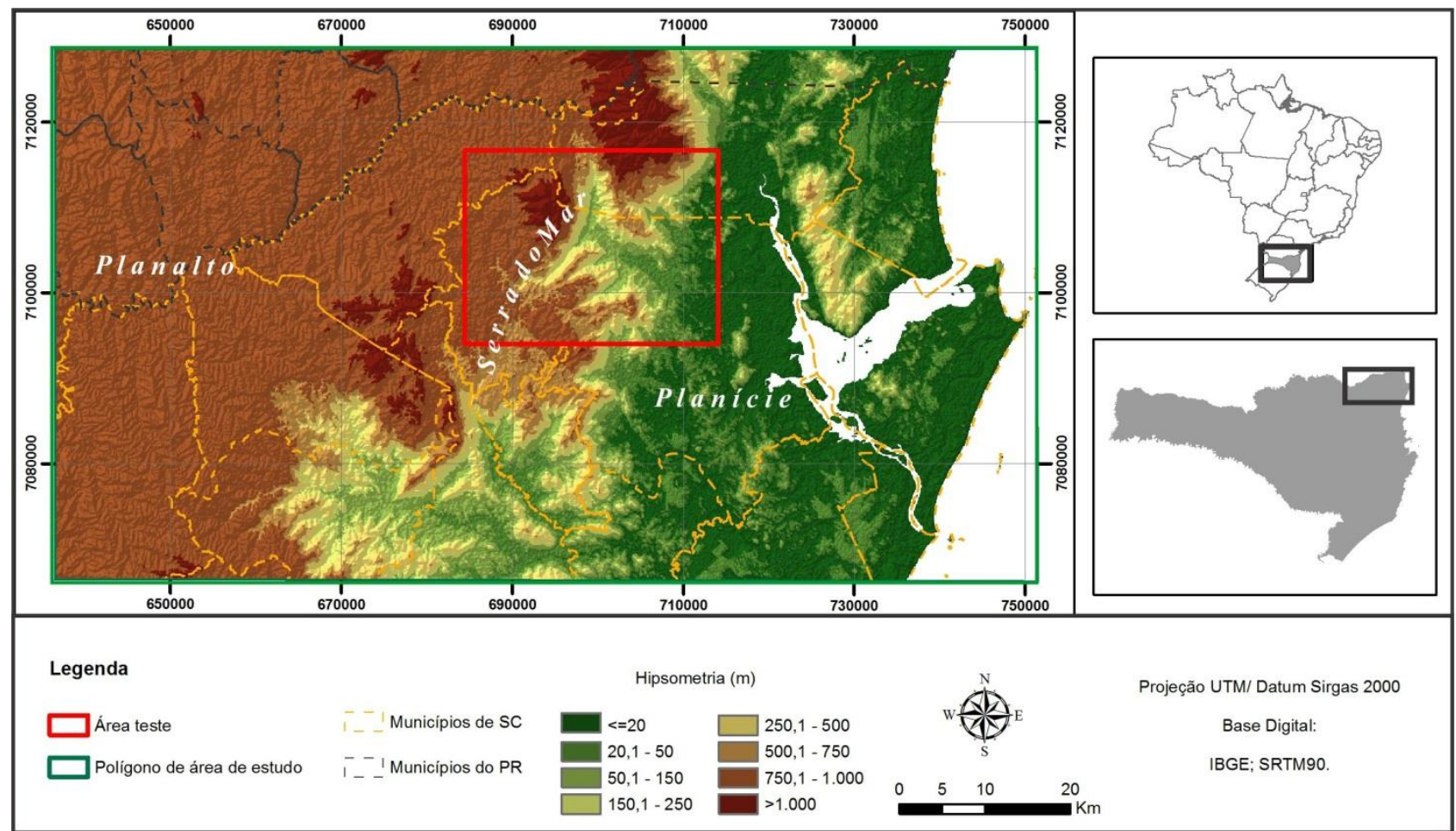

Figura 1. Localização da área de estudo, com destaque para a área teste.

Modelos Digitais de Elevação - MDEs

Quanto à definição, neste trabalho adotouse a conceituação de MDE como aquele que engloba as informações de elevação da superfície (elevação do terreno, e/ou terreno e objetos - como altura de prédios e florestas); MDT, como aquele que se refere apenas aos valores altimétricos do terreno; e MDS, como o modelo que engloba as informações altimétricas e a altura de objetos.

Os arquivos foram trabalhados na projeção UTM - Universal Transversa de Mercator, utilizando o Datum Sirgas 2000. As análises em ambiente de Sistemas de Informação Geográfica (SIG) foram realizadas no software ArcGIS, e as análises estatísticas foram realizadas no software XLSTAT.

Foi selecionada uma área de $670 \mathrm{~km}^{2}$ (aproximadamente 10\% da área de estudo), a qual engloba as diferentes compartimentações topográficas (serra, planalto e planície), para testar a qualidade dos modelos. Esta área foi denominada de "área teste" (Figura 1). Os valores de altimetria dos MDEs foram avaliados em relação a 30 marcos geodésicos (com precisão decimétrica), disponíveis em: https://simgeo.joinville.sc.gov.br/, por meio da geração de estatísticas descritivas e cálculo do Erro Médio Quadrático (EQM) - que se deu por meio da seguinte equação:

$E M Q=\sqrt{\frac{\sum\left(Z_{-} i-Z_{-} t\right)^{2}}{n}}$

Mello, Y. R.; Silveira, C. T.
Onde $Z_{i}$ é o valor altimétrico da célula, $Z_{t}$ é o valor do ponto de controle e $n$ é o número total de pontos de controle.

\section{Modelos Globais}

Os MDEs globais utilizados neste estudo foram os seguintes:

- SRTM90, banda C, versão 4, resolução de 3 arcos de segundo aproximadamente 90 metros. Disponível em: http://srtm.csi.cgiar.org/SELECTION/listImages.a sp;

- $\quad$ SRTM30, banda C, versão 1 , resolução de 1 arco de segundo - aproximadamente 30 metros. Disponível em: http://earthexplorer.usgs.gov/;

- Aster GDEM - Global Digital Elevation Model, versão 2, com resolução de 30 metros. Disponível em: http://earthexplorer.usgs.gov/;

Topodata, MDE com resolução reamostrada para 30 metros, pelo método de krigagem, a partir dos dados SRTM90. Disponível em: http://www.webmapit.com.br/inpe/topodata/.

\section{MDT do Levantamento Aerofotogramétrico de Santa Catarina}

Foram utilizados dados do MDT do levantamento Aerofotogramétrico de Santa Catarina realizado no ano de 2010, disponível no 
site da Secretaria de Desenvolvimento Sustentável de Santa Catarina (SDS) (http://sigsc.sds.sc.gov.br/).

O mosaico dos dados matriciais foi realizado no software ArcGIS, por meio da ferramenta Mosaic Dataset. Os dados originais estão na resolução horizontal de $1 \mathrm{~m}$, e foram exportados para as resoluções de $5 \mathrm{~m}$ e $10 \mathrm{~m}$, devido à capacidade de hardware. Desta forma, foram usadas três resoluções horizontais do mesmo MDT nas análises ( $1 \mathrm{~m}, 5 \mathrm{~m}, 10 \mathrm{~m})$.

\section{Análise de Atributos Topográficos}

Posteriormente as análises preliminares da qualidade dos modelos digitais de elevação na área teste, foram gerados os atributos topográficos primários: declividade (medida de inclinação da superfície), aspecto (orientação das vertentes), plano de curvatura (caráter divergente - vales, convergente - interflúvios, ou planar, dos fluxos de matéria e energia nas vertentes) e perfil de curvatura (forma da vertente: convexa, côncava ou retilínea), ainda na área teste. Na sequência, foram realizadas conferências in loco (as conferências foram realizadas em nove pontos pré-definidos em ambiente SIG, nas três compartimentações topográficas: serra, planalto e planície) com o auxílio de um aparelho GPS (Global Positioning System) Etrex20. Foram avaliados os parâmetros: aspecto, plano e perfil de curvatura e declividade. O trecho de planalto com acesso na área teste é uma transição entre serra e planalto.

A classificação da declividade foi baseada em Embrapa (2006), a qual está organizada nos intervalos entre $0-3 \%$ (relevo plano), $3-8 \%$ (relevo suave ondulado), $8-20 \%$ (relevo ondulado), $20-45 \%$ (relevo forte ondulado), $45-$ $75 \%$ (relevo montanhoso) e > 75\% (relevo escarpado).

Após a verificação do melhor MDE, foram gerados mapas de atributos topográficos primários (hipsometria - discretização da elevação em classes, declividade, aspecto, plano de curvatura, perfil de curvatura e relevo sombreado) e do atributo secundário (Índice de Posição Topográfica - IPT) para toda a área de estudo.

O Índice de Posição Topográfica é uma proposta de classificação geomorfométrica que busca identificar feições e unidades do relevo por meio da combinação de duas escalas de análise: generalizada e detalhada (Jenness, 2004; Tagil e Jenness, 2008; Silveira \& Silveira, 2016 e 2017). O IPT foi elaborado utilizando duas matrizes com variação no raio de análise da vizinhança. Foram testados os raios detalhados de $35 \mathrm{~m}$ (7 pixels) e $105 \mathrm{~m}$ (21 pixels), e os generalizados de $335 \mathrm{~m}$ (67 pixels) e $1005 \mathrm{~m}$ (201 pixels), formando os respectivos pares: 7-67 pixels e 21-201 pixels. Os parâmetros utilizados para o desenvolvimento do IPT estão disponíveis na Tabela 1.

Maiores detalhes sobre a geração destes atributos topográficos, como equações utilizadas, podem ser encontradas nos trabalhos de Silveira \& Silveira (2016 e 2017), pois foram utilizados critérios e modo operacional semelhante.

Tabela 1. Classificação do Índice de Posição Topográfica em dez classes e seus respectivos parâmetros de desenvolvimento.

\begin{tabular}{cccc}
\hline Formas do Relevo & $\begin{array}{c}\text { IPT } \\
\text { generalizado }\end{array}$ & $\begin{array}{c}\text { IPT } \\
\text { detalhado }\end{array}$ & Declividade \\
\hline Vales entalhados & $\leq-\sigma$ & $\leq-1 \sigma$ & \\
Vales rasos; drenagens de meia vertente & $>-1 \sigma \mathrm{e}<1 \sigma$ & $\leq-1 \sigma$ & \\
Nascentes; drenagens de alta vertente & $\geq 1 \sigma$ & $\leq-1 \sigma$ \\
Vales em "U" & $\leq-1 \sigma$ & $>-1 \sigma \mathrm{e}<1 \sigma$ & \\
Planos & $>-1 \sigma \mathrm{e}<1 \sigma$ & $>-1 \sigma \mathrm{e}<1 \sigma$ & $\leq 5$ graus \\
Vertentes intermediárias & $>-1 \sigma \mathrm{e}<1 \sigma$ & $>-1 \sigma \mathrm{e}<1 \sigma$ & $>5$ graus \\
Vertentes superiores & $\geq 1 \sigma$ & $>-1 \sigma \mathrm{e}<1$ & \\
Cristas locais; morros em vales & $\leq-1 \sigma$ & $\geq 1 \sigma$ & \\
Cristas intermediárias; morros em áreas planas & $>-1 \sigma \mathrm{e}<1 \sigma$ & $\geq 1 \sigma$ & \\
Cristas elevadas; topos & $\geq 1 \sigma$ & $\geq 1 \sigma$ & \\
\hline
\end{tabular}

\section{Resultados e discussão}


Foram analisados os erros referentes à altimetria dos sete MDEs descritos na metodologia (SRTM90; SRTM30; ASTER30; Topodata30; SDS1; SDS5; SDS10) relativo à área teste com base em 30 marcos geodésicos distribuídos nos diferentes ambientes (serra, planalto e planície). As estatísticas descritivas e o Erro Médio Quadrático (EMQ) estão descritos na Tabela 2.

Tabela 2. Estatísticas descritivas e EMQ dos MDEs em relação aos marcos geodésicos. Em verde estão destacados os melhores resultados referentes a cada linha, e em vermelho os piores resultados.

\begin{tabular}{|c|c|c|c|c|c|c|c|}
\hline & SRTM90 & SRTM30 & ASTER30 & Topodata30 & SDS1 & SDS5 & SDS10 \\
\hline Erro Médio & 2,6 & 3,1 & 8,2 & 2,8 & 2,6 & 2,6 & 2,8 \\
\hline Erro Mínimo & 0,1 & 0,4 & 0,1 & 0,4 & 0,1 & 0,1 & 0,1 \\
\hline Erro Máximo & 14,1 & 14,7 & 19,2 & 9,0 & 10,2 & 9,2 & 10,2 \\
\hline Desvio Padrão & 2,7 & 2,8 & 5,1 & 2,3 & 2,5 & 2,6 & 2,7 \\
\hline Mediana & 1,8 & 2,5 & 7,6 & 1,9 & 1,9 & 1,3 & 1,3 \\
\hline EMQ & 3,2 & 4,2 & 9,6 & 3,6 & 3,6 & 3,6 & 3,6 \\
\hline
\end{tabular}

O MDE ASTER30 foi o que apresentou os piores resultados nas análises descritivas dos erros. Ouerghi et al. (2015) também não encontraram bons resultados com este modelo e Avtar et al. (2015) obtiveram os mais elevados valores de EMQ, que resulta na menor precisão vertical, quando avaliaram o ASTER GDEM com outros MDEs globais.

Desta forma, optou-se por desconsiderar o MDE ASTER30 nas análises posteriores. De forma geral, para os outros MDEs, os valores de erro, desvio padrão, mediana e EMQ foram similares; tendo o MDE SRTM90 e SDS5 apresentado os melhores resultados, conforme Tabela 2.

Os valores altimétricos dos MDEs em relação aos marcos geodésicos foram subestimados em sua maioria, exceto no MDE Topodata30, conforme Tabela 3. O MDE ASTER30 foi o que apresentou a maior frequência de dados subestimados, outros autores na literatura também encontraram maiores frequências subestimadas para este MDE (Rexer et al., 2014; Ouerghi et al., 2015).

Tabela 3. Valores de erros subestimados e superestimados dos MDEs em relação aos marcos geodésicos em (\%).

\begin{tabular}{cccccccc}
\hline & SRTM90 & SRTM30 & ASTER30 & Topodata30 & SDS1 & SDS5 & SDS10 \\
\hline Subestimado & 70,0 & 56,7 & 96,7 & 40,0 & 83,3 & 80,0 & 80,0 \\
Superestimado & 30,0 & 43,3 & 3,3 & 60,0 & 16,7 & 20,0 & 20,0 \\
\hline
\end{tabular}

Atributos Topográficos

Área teste

Foram elaborados os mapas dos atributos topográficos (declividade, aspecto, plano de curvatura e perfil de curvatura) para seis MDEs (SRTM90, SRTM30, Topodata30, SDS10, SDS5,
SDS1), conforme Figura 2. As diferenças foram aumentando gradualmente entre os modelos, quanto maior a amplitude da resolução horizontal de grade. A Figura 3 traz a frequência em (\%) das classes referentes a cada atributo, como auxílio nas discussões seguintes. 

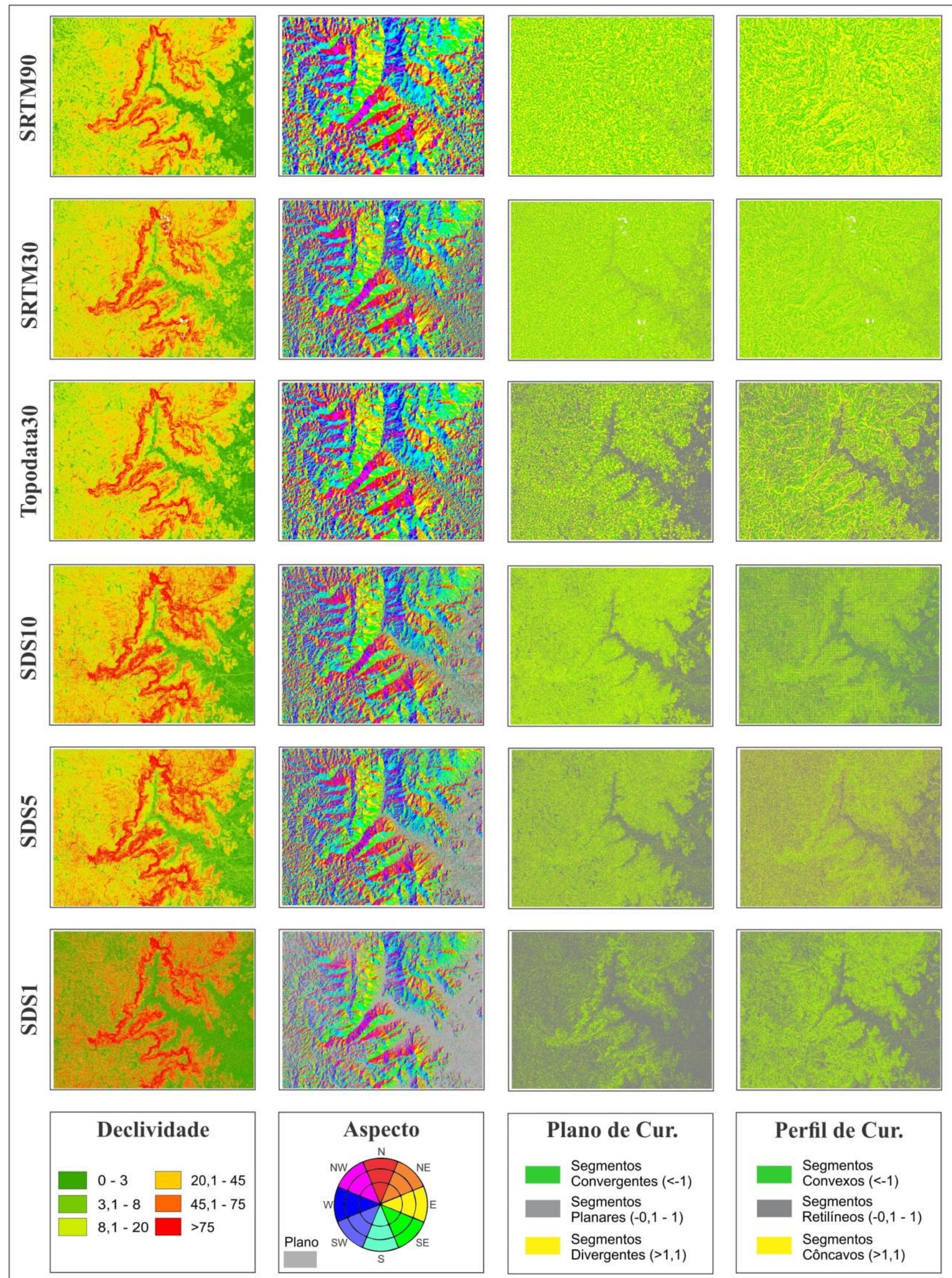

Figura 2. Comparação dos atributos topográficos (declividade, aspecto, plano de curvatura e perfil de curvatura) na área teste, entre diferentes MDEs (SRTM90, SRTM30, Topodata30, SDS10, SDS5 e SDS1).

A declividade foi um atributo que variou muito entre os MDEs. Quanto menor o pixel do MDE, maiores os valores de declividade Mello, Y. R.; Silveira, C. T. encontrados. Por exemplo, o MDE SRTM90 apresentou declividade máxima de 177,3\%, enquanto para o MDE SDS1 o valor máximo foi de 
$3.837,5 \%$. Quanto às frequências de classes (Figura 3), o MDE SDS1 foi o que apresentou as maiores diferenças em relação à média dos MDEs por classe, com destaque para as classes $<3 \%, 20-45 \%$ e $45-75 \%$, que apresentaram diferença média de $20 \%$.

Nesse contexto, Hengl (2006) expressa que, de modo geral, o aumento do detalhe do MDE significa parâmetros do terreno mais precisos. Assim, quando se avalia a declividade, principalmente em vertentes mais complexas, com maior variação da declividade, o resultado é notável.

Neuman et al. (2018), igualmente detectaram uma grande variação na geração da declividade ao utilizar distintos MDEs com resolução variada. Os Autores perceberam que as variações apresentadas foram resultado do tamanho de pixel utilizado em função da escala da base de dados de origem. Perceberam que na medida em que se reduz a resolução dos MDEs, no caso do emprego do SRTM90 e SRTM30, ocorreu no seu experimento a concentração de valores de células de declividade no intervalo entre 8-20\%, representado por valores intermediários. Enquanto que os MDEs de maior resolução, com 1 e 5m, apresentaram valores de declividade menos concentrado no intervalo supra mencionado, com presença destacada de células com valores entre $20-45 \%$ e superior a $45 \%$.

Quanto ao aspecto (orientação das vertentes), o MDE SDS1 foi o que apresentou as maiores diferenças em relação aos outros MDEs, principalmente quanto à classe "Plano", com uma diferença aproximada de $30 \%$ em relação à média. Quanto mais grossa a resolução de grade (tamanho do pixel), pior foi o cenário em relação a esta classe, por exemplo, os MDEs SRTM90 e Topodata30 não apresentaram nenhum valor "Plano", e para o MDE SRTM30, a frequência foi de $0,1 \%$. Sabe-se empiricamente que parte considerável da área teste é plana, o que desfavorece a utilização destes modelos em relação a este atributo. Destaca-se que este atributo é uma ferramenta importante, por exemplo, em uma análise climatológica, pois a orientação das vertentes vai interferir na entrada de fluxos de umidade e de radiação solar.

Tanto o mapa do plano de curvatura, quanto o de perfil de curvatura geraram segmentos planares e retilíneos, respectivamente, contrastantes entre os modelos. Em relação à frequência das classes no plano de curvatura, mais uma vez o MDE SDS1 foi o que apresentou as maiores diferenças em relação à média dos MDEs, categorizando quase $80 \%$ da área teste como planar, enquanto para o MDE SRTM90 o valor foi de apenas $3,4 \%$.

No mapa de perfil de curvatura o MDE SRTM90 foi o que apresentou as maiores diferenças em relação a médias dos modelos, novamente com um baixo valor de segmentos retilíneos. A maior diferença entre os segmentos convexos e côncavos se deu no MDE SRTM90 $(12,2 \%)$. Para este atributo, a diferença entre o segmento retilíneo e os outros não foi tão contrastante no SDS1.

No caso dos atributos de perfil e plano de curvatura, Neuman et al. (2018) observou que o efeito do aumento no tamanho do pixel, diminuição da resolução, observando distintos MDEs em seu experimento, resultou em expressivo aumento das classes retilíneas e planares, principalmente no SRTM90 e SRTM30. Fato igualmente identificado nas observações do presente trabalho.

Foram realizadas observações in loco para validar a qualidade dos modelos. Verificou-se se os atributos primários (declividade, aspecto, plano de curvatura e perfil de curvatura) gerados em ambiente SIG, a partir dos seis diferentes MDEs, condiziam com a realidade, por meio de nove amostras selecionadas nos três ambientes (serra, planalto e planície) (Figura 3). 


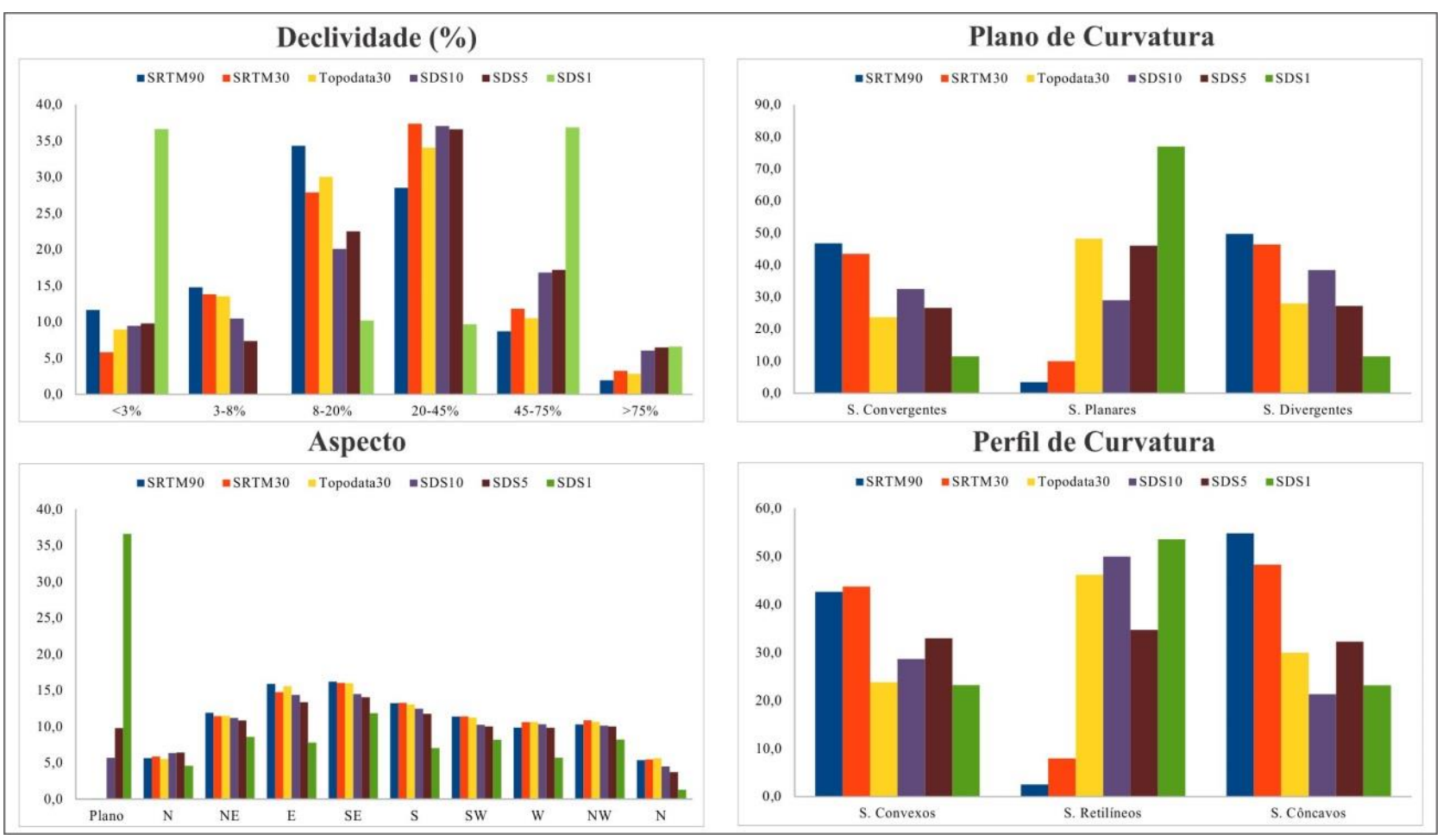

Figura 3. Gráficos comparativos da frequência das classes dos atributos topográficos (declividade, aspecto, plano de curvatura e perfil de curvatura) na área teste, entre diferentes MDEs (SRTM90, SRTM30, Topodata30, SDS10, SDS5 e SDS1). 

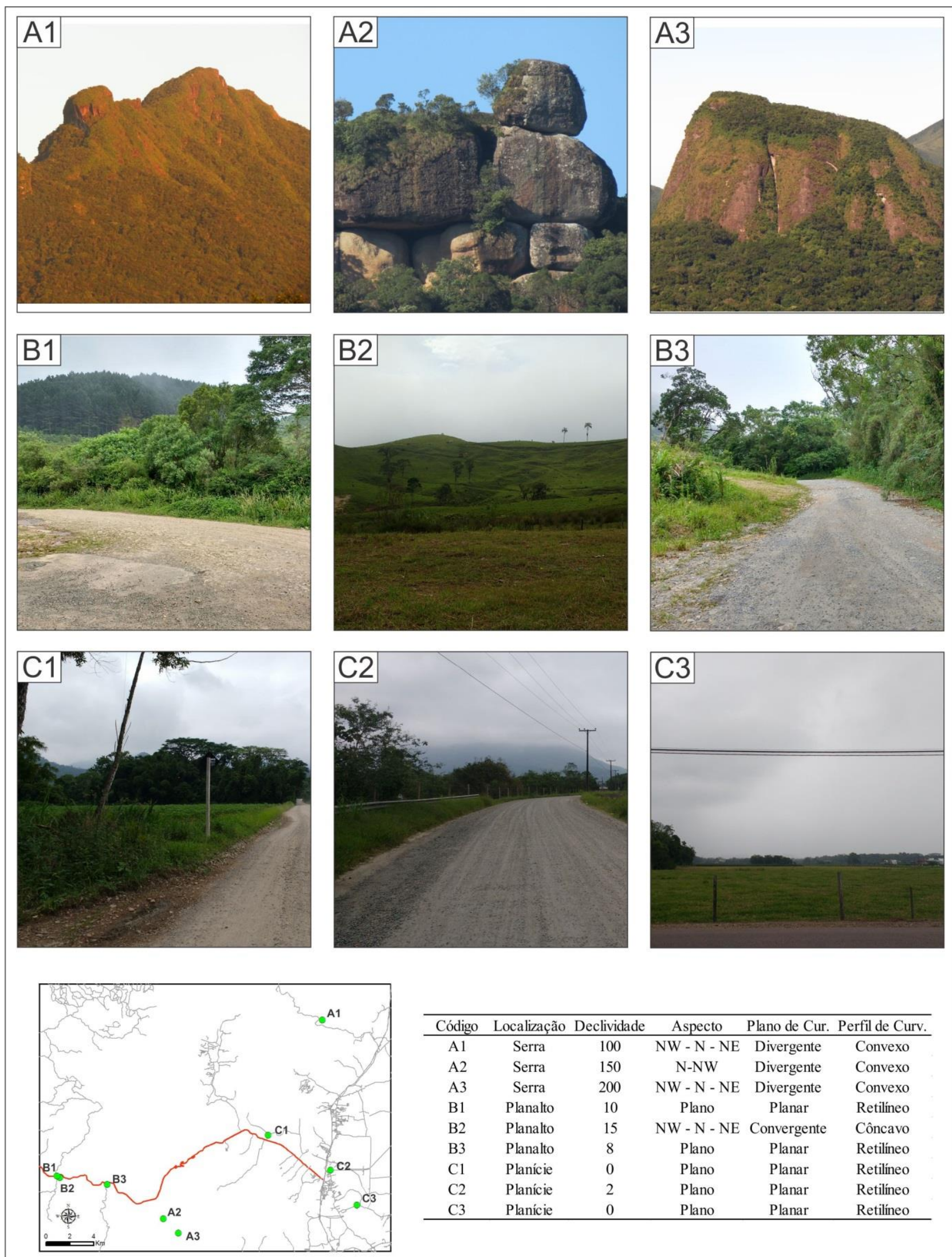

\begin{tabular}{cccccc}
\hline Código & Localização & Declividade & Aspecto & \multicolumn{2}{c}{ Plano de Cur. Perfil de Curv. } \\
\hline A1 & Serra & 100 & NW - N - NE & Divergente & Convexo \\
A2 & Serra & 150 & N-NW & Divergente & Convexo \\
A3 & Serra & 200 & NW - N - NE & Divergente & Convexo \\
B1 & Planalto & 10 & Plano & Planar & Retilíneo \\
B2 & Planalto & 15 & NW - N - NE & Convergente & Côncavo \\
B3 & Planalto & 8 & Plano & Planar & Retilíneo \\
C1 & Planície & 0 & Plano & Planar & Retilíneo \\
C2 & Planície & 2 & Plano & Planar & Retilíneo \\
C3 & Planície & 0 & Plano & Planar & Retilíneo \\
\hline
\end{tabular}

Figura 4. Pontos de conferência de campo.

O MDE SDS1 se destacou nas comparações em relação ao ambiente da planície, sendo o melhor modelo, seguido pelo Topodata30. Já os MDEs SRTM90 e SRTM30 apresentaram

Mello, Y. R.; Silveira, C. T. resultados ruins no ambiente de planície, o que também é evidenciado nas Figuras 2 e 3 . Em relação ao ambiente de serra o SDS5 apresentou o maior número de acertos em relação ao real; já no ambiente de planalto foi o SDS1. Todos os 
modelos, exceto o SDS1, apresentaram o maior número de acertos no ambiente serra. Já em relação aos atributos, a declividade foi melhor representada pelo SDS10, o aspecto pelo SDS1, o plano de curvatura pelo SDS1 e SDS10, e o perfil de curvatura pelo SDS1 e Topodata30.

Com base nas análises dos atributos topográficos e nas conferências em campo, ficou evidenciado que os piores modelos foram o SRTM30 e o SRTM90, e os melhores foram o SDS1, SDS5 e SDS10. Os modelos SRTM30 e SRTM90 apresentaram resultados insatisfatórios em relação aos atributos aspecto, plano de curvatura e perfil de curvatura, com uma má representação de áreas planas/retilíneas. Apesar disso, todos os modelos apresentaram bons resultados em diferentes análises, por exemplo, o SRTM90 apresentou o menor EMQ. Optou-se por utilizar o MDE SDS5 - que apresentou resultados similares ao SDS10, porém maior detalhamento porque apesar de o SDS1 apresentar bons resultados e maior detalhamento, o polígono de área de estudo possui uma área aproximada de $7.000 \mathrm{~km}^{2}$, e o processamento dos dados se tornou inviável devido à capacidade de hardware.

\section{Polígono de área de estudo (área total)}

Foram gerados os mapas dos atributos topográficos primários: hipsometria, declividade, aspecto (Figura 5), plano de curvatura, perfil de curvatura, relevo sombreado (Figura 6); e do atributo topográfico secundário Índice de Posição Topográfica (Figura 7), para toda a área de estudo. A Figura 8 traz as frequências das classes encontradas nos atributos como forma de auxiliar nas análises imediatas.

O valor altimétrico médio da área de estudo é de $447 \mathrm{~m}$, o qual varia do nível do mar até $1.540 \mathrm{~m}$. A classe predominante é a de 750,1 a 1000 $\mathrm{m}(36,1 \%)$, correspondente principalmente à região de planalto. As áreas planas inferiores a 20,1 m são em sequência as mais frequentes $(29,9 \%)$. As áreas com altitudes superiores a 1.000 metros estão distribuídas pela Serra do Mar, no Granito Morro Redondo, Granito Dona Francisca, Granito Piraí, Granito Corupá e um pouco mais a oeste em coberturas vulcanossedimentares da Formação Campo Alegre (CPRM, 2014).
Quanto à declividade, o valor médio foi de $21,4 \%$, variando de 0 a $10.074,9 \%$. As classes predominantes são a de 20 a $45 \% /$ relevo forte ondulado com $27,1 \%$ de frequência e de 8 a $20 \% /$ relevo ondulado com $26,8 \%$ de frequência. As maiores declividades são encontradas na escarpa da Serra do Mar, na porção central da área de estudo, o relevo escarpado corresponde a 2,9\% da área total analisada, e o relevo montanhoso corresponde a $10,3 \%$.

Para o atributo aspecto (orientação das vertentes) a classe predominante foi o plano $(22,4 \%)$, seguido por leste $(10,5 \%)$, sudeste $(10,4 \%)$ e nordeste $(10 \%)$. A orientação com a menor frequência é a de sudoeste (9\%), ou seja, a diferença entre a orientação das vertentes é mínima, já que a amplitude entre as direções leste e sudoeste é de apenas 1,5\%. A classe "plano" predomina nas áreas de planície costeira. Na região de planalto a variabilidade entre as diferentes orientações é mais homogênea, devido à característica do relevo (pequenas cristas, morros de topos convexos e vales em "V" e simétricos). Já na área compreendida pelas escarpas da Serra do Mar há um predomínio da orientação leste, sudeste e nordeste, com destaque para as encostas à leste dos granitos Morro Redondo e Dona Francisca. Segundo Almeida e Carneiro (1998) a direção geral da Serra do Mar acompanha a orientação leste/nordeste do Escudo Atlântico, justificando estes resultados.

Os atributos: plano de curvatura e perfil de curvatura estão associados aos processos erosivos do relevo. Os valores médios desses dois atributos foram equivalentes a 0 , indicando que a área de estudo têm predominância de segmentos planares e retilíneos, o que é comprovado no gráfico de frequência na Figura 8, onde os segmentos planares representam $57,7 \%$ e os segmentos retilíneos $44,3 \%$. O atributo relevo sombreado, por sua vez, é uma importante ferramenta na interpretação visual das formas do relevo. Este atributo possibilita analisar o sombreamento resultante das formas do relevo, o qual indica as áreas que recebem maior ou menor radiação solar. $\mathrm{O}$ que pode ser útil em análises climatológicas. 

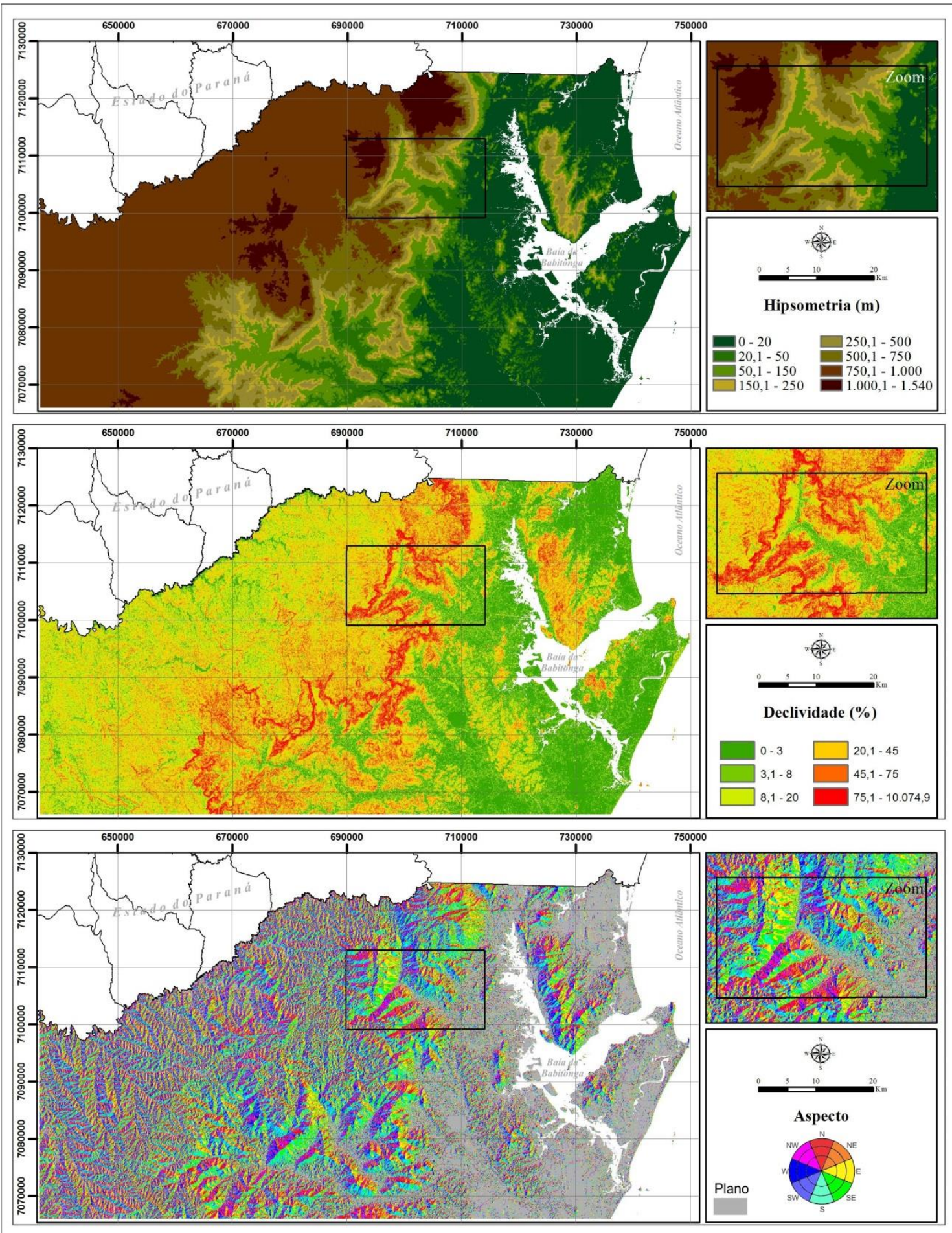

Figura 5. Atributos topográficos primários (hipsometria, declividade e aspecto) da região da Serra do Mar do estado de Santa Catarina. 
Revista Brasileira de Geografia Física v.11, n.06 (2018) 2029-2045.
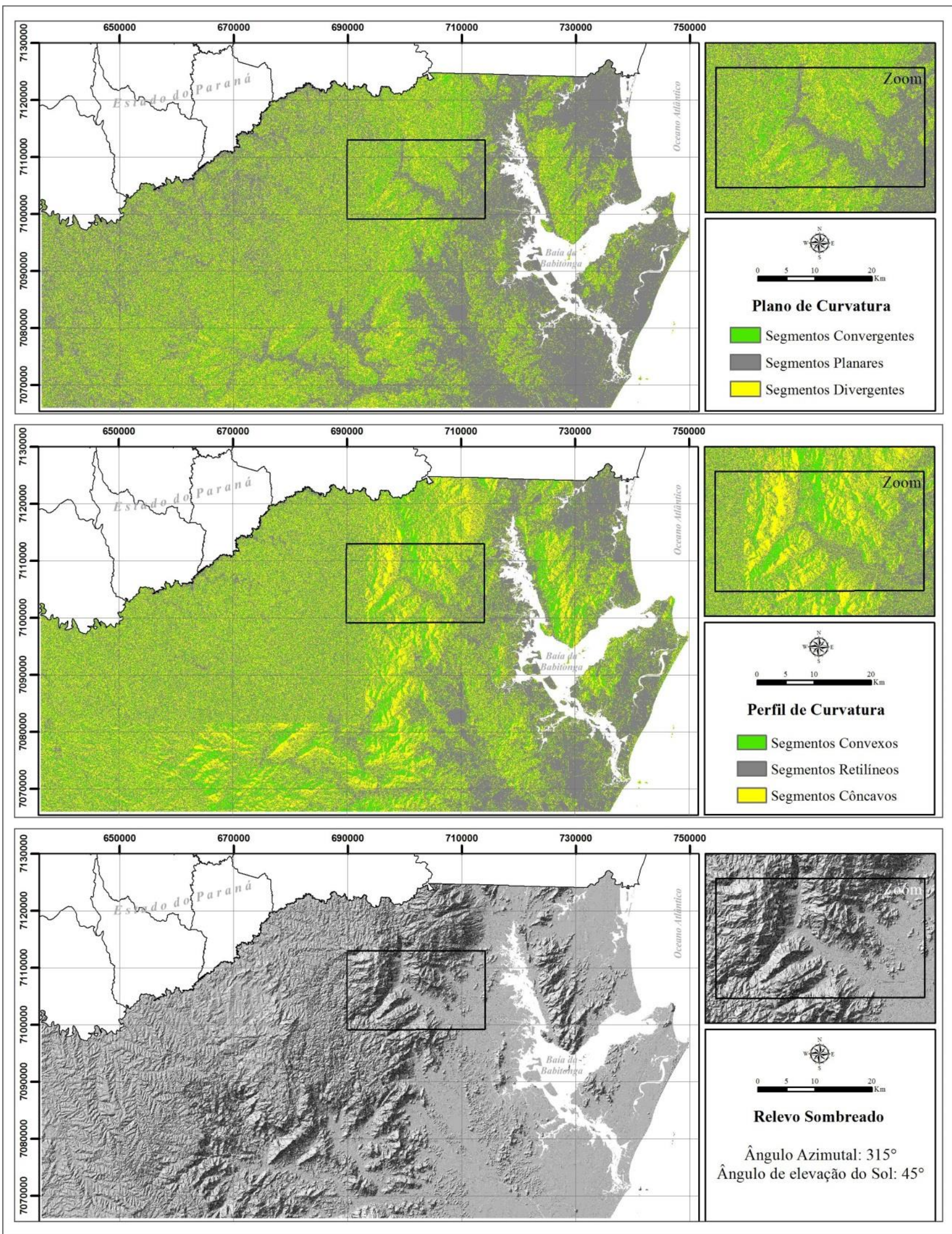

Figura 6. Atributos topográficos primários (plano de curvatura, perfil de curvatura e relevo sombreado) da região da Serra do Mar do estado de Santa Catarina. 


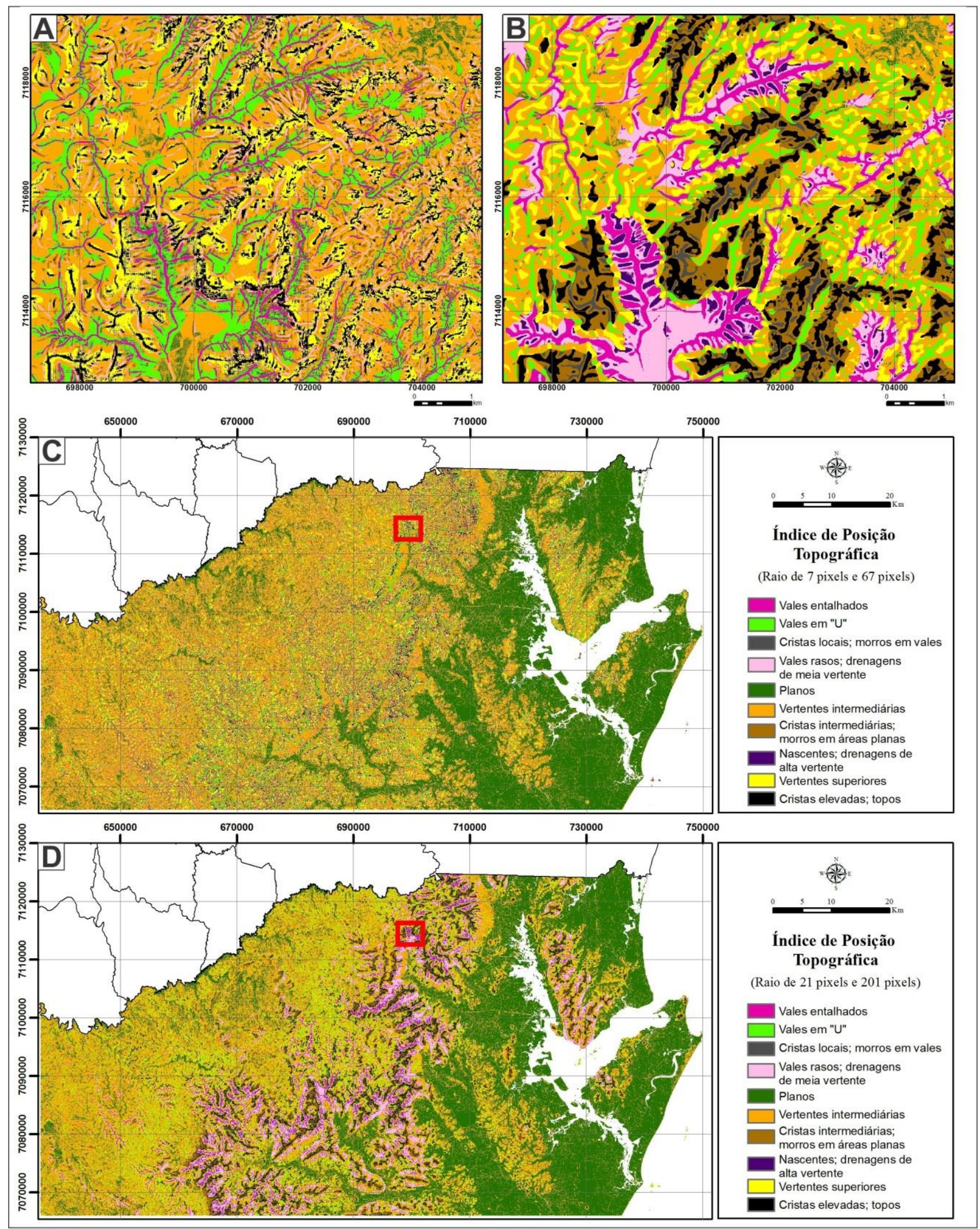

Figura 7. A) Área destaque do IPT (raio de 7 e 67 pixels); B) Área destaque do IPT (raio de 21 e 201 pixels); C) IPT (raio de 7 e 67 pixels) para a área de estudo; D) IPT (raio de 21 e 201 pixels) para a área de estudo. 

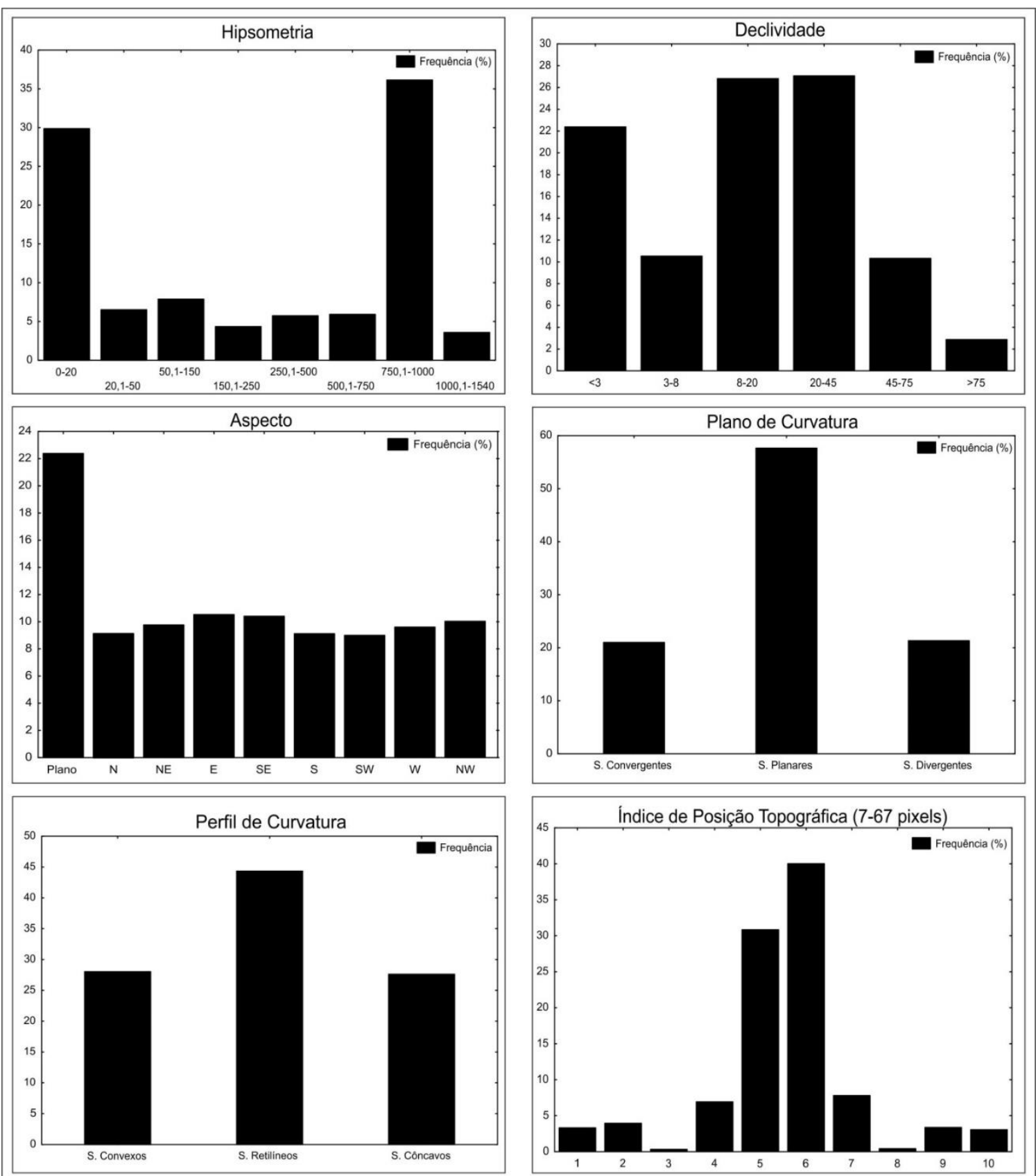

Figura 8. Frequência de classes de atributos topográficos.

Ambos os pares de análise do IPT: 7-67 pixels e 21-201 pixels apresentaram resultados satisfatórios, porém o IPT com a menor janela amostral (7-67 pixels) apresentou resultados mais fidedignos (Figura 8). Silveira \& Silveira (2016) encontraram resultados semelhantes estudando a porção central da Serra do Mar do estado do Paraná. O IPT com a maior janela amostral generalizou as classes gerando exageros desproporcionais, por exemplo, uma crista localizada na porção nordeste da área de estudo (município de Garuva) com largura aproximada de 30 metros, foi categorizada com largura aproximada de 100 metros no IPT 21-201 pixels. Essa dinâmica de generalização das classes no IPT com a maior janela amostral também pode ser observada na Figura 7, onde foram selecionadas duas áreas menores (mais detalhadas) - destacadas em vermelho nos mapas de IPT da área de estudo (Figura 7C-D), na Figura 7A foi destacado o IPT (7-67 pixels) e na Figura 7B o IPT (21-201 pixels). Na Tabela 4 estão descritas as frequências de cada classe do IPT para as duas janelas amostrais, com base na tabela é possível observar que a frequência das classes foi similar entre os dois modelos. As maiores diferenças foram observadas na classe de vertentes intermediárias. A classe predominante na área de estudo (considerando o IPT 7-67 pixels) é a de vertentes intermediárias, seguida pela classe de planos $(30,8 \%)$. 
Tabela 4. Frequência das classes do Índice de Posição Topográfica para as duas janelas analisadas para a região da Serra do Mar de Santa Catarina.

\begin{tabular}{cccc}
\hline & Formas do Relevo & Frequência IPT & $\begin{array}{c}\text { Frequência } \\
\text { IPT (21- } \\
\text { 201 pixels })\end{array}$ \\
\hline 11 & Vales entalhados & 3,3 & 3,1 \\
22 & Vales rasos; drenagens de meia vertente & 3,9 & 5,3 \\
33 & Nascentes; drenagens de alta vertente & 0,3 & 0,5 \\
44 & Vales em "U" & 6,9 & 7,2 \\
55 & Planos & 30,8 & 30,4 \\
66 & Vertentes intermediárias & 40,0 & 37,3 \\
77 & Vertentes superiores & 7,8 & 6,5 \\
88 & Cristas locais; morros em vales & 0,4 & 0,7 \\
99 & Cristas intermediárias; morros em áreas planas & 3,4 & 4,8 \\
110 & Cristas elevadas; topos & 3,1 & 4 \\
\hline
\end{tabular}

\section{Conclusões}

O MDE SDS5 se mostrou o modelo mais adequado para o estudo, porém este material está disponível apenas no estado de Santa Catarina. Os modelos globais apresentaram-se insatisfatórios em relação aos atributos topográficos: declividade, aspecto, plano de curvatura e perfil de curvatura. Para o atributo declividade os modelos com as resoluções de grade mais grosseira apresentaram valores baixos em áreas de serra, se comparado ao MDE de Santa Catarina. Para os atributos aspecto, plano e perfil de curvatura os MDEs globais não representaram as áreas planas com acurácia. $\mathrm{O}$ MDE ASTER 30 apresentou os piores resultados já na validação dos dados em relação aos pontos de controle. De todo modo, em muitos casos de estudo os modelos globais são a única opção, e podem sim trazer importantes contribuições para o trabalho, porém, de forma mais generalizada.

Os atributos topográficos primários: hipsometria, declividade, aspecto, perfil de curvatura, plano de curvatura e relevo sombreado; e o atributo secundário Índice de Posição Topográfica, gerados para toda a área de estudo pelo MDE SDS5, se mostraram importantes ferramentas para melhor compreender o relevo regional.

\section{Agradecimentos}

A primeira autora agradece ao Programa de Pós-Graduação em Geografia da UFPR pela concessão de bolsa Capes de doutorado.

\section{Referências}

Almeida, F. F. M. de, Carneiro, C. D. R. 1998. Origem e evolução da Serra do Mar. Revista Brasileira de Geociências 28, 135-150.

Avtar, R.; Yunusb, A.P.; Krainesb, S.; Yamamurob, M. 2015. Evaluation of DEM generation based on Interferometric SAR using TanDEM-X data in Tokyo. Physics and Chemistry of the Earth, v. 83-84, 166-177.

CPRM. Serviço Geológico do Brasil, 2014. Mapa Geológico do estado de Santa Catarina. Porto Alegre.

De Reu, J.; Bourgeoisa, J.; Batsa, M.; Zwertvaegherb, A.; Gelorinib, V.; Smedtc, P.; Chue, W.; Antropd, M.; Maeyerd, P.; Finkeb, P.; Meirvennec, M.; Verniersb, J.; Crombéa, P. 2013. Application of the topographic position index to heterogeneous landscapes. Geomorphology, 186, 39-49.

EMBRAPA. Empresa Brasileira e Pesquisa Agropecuária, 2006. Sistema Brasileiro de Classificação de Solos. Brasília.

Fan, F. M., Collischonn, W., Sorribas, M. V., Pontes, P. R. M. 2013. Sobre o Início da Rede de Drenagem Definida a Partir dos Modelos 
Digitais de Elevação. Revista Brasileira de Recursos Hídricos 18, 241-257.

Gomes, S. M. A.; Silveira, R. M. P.; Silveira, C. T. 2018. Aplicação de técnicas geomorfométricas para classificação de formas do relevo em Campo Largo, estado do Paraná - Brasil. Revista Geografar, 13, 1, 75-97.

Guadagnin, P.M.A.; Trentin, R. 2014. Compartimentação geomorfométrica da bacia hidrográfica do Arroio Caverá - RS. Geo UERJ, 16, , 1, 25, 183-199.

Hengl, T. 2006. Finding the right pixel size. Computers \& Geosciences, 32, 1283-1298.

Hengl, T., Reuter, H. I. 2009. Geomorfometry: concepts, software, applications. Elsevier, Amsterdam.

IBGE. Instituto Brasileiro de Geografia e Estatística,2016. Cidades@. Disponível em: http://www.cidades.ibge.gov.br. Acesso: 06 dez. 2016.

Jenness, J. S. 2004. Calculating landscape surface area from digital elevation models. Wildlife Society Bulletin 32, 829-839.

Neuman, G.; Silveira, C.T.; Sampaio, T.V. 2018. Análise da influência da escala na obtenção dos atributos topográficos derivados de MDE. Revista Ra'e Ga, 43, 179 -199.

Nowatzki, A; Santos, L.J.C; Silveira, C.T. 2017. Potencialidade do Índice de Posição Topográfica no Mapeamento Pedológico Preditivo em uma Sub-Bacia do Rio Capricórnio-PR. Revista do Departamento de Geografia, 34, 10-20.

Oliveira, F. A. de. 2006. Estudo do aporte sedimentar em suspensão na baía da babitonga sob a ótica da geomorfologia. Tese (Doutorado). São Paulo, Universidade de São Paulo - USP.

Ouerghi, S., Elsheikh, R. F. A., Achour, H., Bouazi, S. 2015. Evaluation and Validation of Recent Freely-Available ASTER-GDEM V.2, SRTM V.4.1 and the DEM Derived from Topographical Map over SW Grombalia (Test Area) in North East of Tunisia. Journal of Geographic Information System 7, 266-279.

Pike, R. J. 2000. Geomorphometry - diversity in quantitative surface analysis. Progress in Physical Geography 24, 1, 1-20.

Pozzobon, M; Curcio, G.R; Silveira, C.T. 2015. Análise da suscetibilidade a deslizamentos da unidade geomorfológica Serrania do Baixo e Médio Itajaí-açu, no município de Blumenau/SC. Revista Brasileira de Geologia de Engenharia e Ambiental, 5, 2, 9-25.

Rexer, M., Hirt, C. 2014. Comparison of free highresolution digital elevation data sets (ASTER GDEM2, 11 SRTM v2.1/v4.1) and validation against accurate heights from the Australian 12 National Gravity Database. Australian Journal of Earth Sciences, 1-15.

Silva, J. M. F.; Oka-Fiori, C.; Silveira, C. T. 2017a. Geomorfometria: uma análise de conceitos, métodos e aplicações em geografia física. Revista Brasileira de Geografia Física, 10, 2, 558-583.

Silva, J. M. F.; Oka-Fiori, C.; Silveira, C. T. 2017b. Índice de Diversidade de Formas de Relevo do Setor Norte da Apa da Serra da Esperança (PR) com Emprego de Técnica de Classificação Geomorfométrica. Revista do Departamento de Geografia, Volume Especial,112-123.

Silveira, C. T. Oka-Fiori, C.; Santos, L. J. C; Sirtoli, A. E.; Silva, C. R.; Botelho, M. F. 2013. Soil prediction using artificial neural networks and topographic attributes. Revista Geoderma, 195-196, 165-172.

Silveira, C.T; Fiori, A.P; Ferreira, A.M; Góis, J.R; Mio, G; Silveira, R.M.P; Massulini, N.E.B; Leonardi, T.M.H. 2013. Emprego de atributos topográficos no mapeamento da susceptibilidade a processos geoambientais na bacia do rio Jacareí, Paraná. Sociedade \& Natureza, 25, 3, 623-639.

Silveira, C.T; Fiori, A.P; Schilipack,P; Dias, S.M. 2014. Mapeamento preliminar da suscetibilidade natural a movimentos de massa da Serra do Mar Paranaense apoiado na análise digital do relevo. Revista Brasileira de Geomorfologia, 15, 1, p.3-22

Silveira, C. T.; Silveira, R. M. P. 2017. Índice de Posição Topográfica (IPT) para classificação geomorfométrica das formas de relevo no estado do Paraná - Brasil. Revista Ra'e Ga, v. 41, 98-130.

Silveira, R. M. P.; Silveira, C. T.; Oka-Fiori, C. 2014. Emprego de técnicas de inferência espacial para identificação de unidades de relevo apoiado em atributos topográficos e árvore de decisão. Revista Brasileira de Geomorfologia, 15, 1, 87-101.

Silveira, R. M. P., Silveira, C. T. da. 2015. Análise comparativa entre Modelos Digitais de Elevação com distintas características de processamento e aquisição. Boletim de Geografia, Maringá 33, 106-121.

Silveira, R. M. P.; Silveira, C. T. 2016. Análise digital do relevo aplicada à cartografia geomorfológica da porção central da Serra do Mar Paranaense. Revista Brasileira de Geomorfologia, 17, 4, 615-629.

Tagil, S. \& J.S. Jenness. 2008. GIS-based automated landform classification and topographic, landcover and geologic attributes 
of landforms around the Yazoren Polje, Turkey. Journal of Applied Sciences 8, 910-921.

Tinós, T.M.; Ferreira, V.V.; Riedel, P.S.; Zaine, J.E. 2014. Aplicação e avaliação de metodologia de classificação automática de padrões de formas semelhantes do relevo.
Revista Brasileira de Geomorfologia, 15, 3, 353-370.

Trentin, R.; Robaina, L. E. S.; Silveira, C. T. 2015. Compartimentação geomorfométrica da bacia Hidrográfica do rio Itú/RS. Revista Brasileira de Geomorfologia, 16, 2, 219-237. 